\title{
La formation en radioprotection du personnel des centrales nucléaires d'Électricité de France
}

\author{
Louis AYE ( $\left.{ }^{1}\right)$ \\ (Manuscrit reçu le 6 juillet 1980)
}

\begin{abstract}
RÉSUMÉ
La sécurité du personnel et du public est certainement aujourd'hui la préoccupation majeure de l'industrie nucléaire. Pour mener à bien son programme de centrales PWR, Électricité de France a mis l'accent sur la formation en radioprotection de son personnel.

Les agents d'exploitation, qui représentent actuellement environ 5000 personnes, reçoivent une formation, formalisée au niveau national, sous forme de stages et de chantiers-écoles, puis assurée et entretenue " sur le tas ». Cette formation fait largement appel aux techniques pédagogiques audiovisuelles, elle est contrôlée par des tests et donne lieu à des habilitations.

Une concertation étroite est souhaitée avec les services compétents extérieurs, ceux du C.E.A., du S.C.P.R.I., ou des centres professionnels de formation.
\end{abstract}

\begin{abstract}
The safety of workers and the population is a major concern of the nuclear industry. In order to carry out its programme of PWR power plants, Électricité de France has largely developed the training in radiation protection of its personnel. Operation workers now represent some 5000 persons; they first receive a formation organized at the national level consisting in training courses, which are completed and continued on the spot. The training makes a wide use of audiovisuals; it is checked by tests and leads to better qualification. Close coordination is sought with outside competent organizations.
\end{abstract}

\section{INTRODUCTION}

La radioprotection est une des branches de la sécurité des travailleurs et du public. Elle commence donc au niveau du projet de réalisation de toute

(*) Électricité de France, Service de la Production thermique, Département de Radioprotection, B.P. $\mathrm{n}^{\circ} 114,93203$ Saint-Denis.

RADIOPROTECTION, VOL. 15 - 0033-8451/1980/183/\$ 5.00/C) Bordas-Dunod. 
installation nouvelle et concerne tous les partenaires impliqués dans cette réalisation, ingénieurs, techniciens, médecins, contrôleurs de l'État, etc.

Pour que chacun ait bien le souci permanent de la réduction des doses et des expositions, il est nécessaire de lui fournir, par une formation appropriée, le niveau de compétence souhaitable.

Dans le cas des centrales nucléaires, E.D.F. se trouve confronté à un double problème :

- la réalisation d'un programme d'équipement important sur une période relativement courte;

- le passage au stade de la série industrielle de la filière de réacteurs à eau pressurisée (PWR) qui, malheureusement, présente un certain nombre d'inconvénients au plan de la radioprotection par rapport aux autres filières adoptées en France (graphite-gaz et surgénérateurs).

Ces deux facteurs pourraient conduire à un relâchement de l'attention et des efforts déployés en matière de sécurité du personnel. Nous allons tenter de montrer qu'il n'en est rien et qu'en particulier le Service de la Production thermique met en œuvre un plan de formation important.

Ce service, actuellement de 15000 personnes, dispose de 5000 agents DATR $\left({ }^{1}\right)$ répartis dans 45 tranches en service ou en essais de démarrage. Cet effectif est d'ailleurs en rapide augmentation d'environ 700 agents par an, comme l'indique le tableau I.

TABLEAU I

ÉVOLUTION APPROXIMATIVE DES EFFECTIFS D.A.T.R. du Service de la Production ThermiQue E.D.F.

\begin{tabular}{|c|c|c|c|c|c|c|}
\hline 1979 & 1980 & 1981 & 1982 & 1983 & 1984 & 1985 \\
\hline 3400 & 4400 & 5100 & 5900 & 6600 & 7300 & 8100 \\
\hline
\end{tabular}

E.D.F. s'est également préoccupé de la formation d'agents d'entreprises extérieures qui constituent, notamment pendant les périodes d'arrêt pour entretien des tranches PWR, un renfort notable et nécessaire aux effectifs de la centrale.

\section{LES PRINCIPES DE BASE DE LA FORMATION EN RADIOPROTECTION A E.D.F.}

\subsection{PRINCIPES ESSENTIELS}

\section{Pourquoi former?}

Si les risques dus aux rayonnements ionisants présentent de nombreuses similitudes avec les autres risques encourus dans une centrale thermique (risques dus à l'utilisation de la chaleur, de l'électricité, des produits chimiques, etc.) ils ont néanmoins, à leur détriment, deux caractéristiques qu'il convient ici de rappeler :

(1) Directement affectés à des travaux sous rayonnements. 
- ils ne sont pas perceptibles par nos sens;

- ils restent, de la majorité des travailleurs et, a fortiori, de la population, inconnus ou mal connus.

Le premier point ne devrait pas représenter un obstacle insurmontable; l'expérience montre, en effet, qu'on apprend à se protéger contre d'autres risques « invisibles » ou " inodores », dans le domaine des substances chimiques toxiques par exemple.

Par contre, la mauvaise appréciation des risques nucléaires est plus délicate à surmonter. L'enseignement de l'énergie nucléaire dès l'école secondaire ou technique contribuera à familiariser les jeunes avec la radioactivité et les notions de curie et de rem (et, a fortiori, de gray et de sievert). Par ailleurs, on peut espérer une attitude plus rationnelle du public et des mass media vis-à-vis de l'énergie nucléaire et de ses véritables risques.

En attendant, l'employeur doit se substituer pour une bonne part à l'éducation nationale et combler les lacunes de l'enseignement général en assurant à ses propres agents le niveau de base indispensable. Il doit répondre aussi aux inquiétudes des agents récemment recrutés, qui sont les mêmes que celles de la population.

\section{Qui former en radioprotection?}

La prise de conscience permanente est indispensable si l'on veut, dans uns installation industrielle, réduire les doses à un niveau " aussi bas que raisonnablement possible ", compte tenu des contraintes économiques.

Le travail en zone contrôlée présente de nombreuses similitudes avec les « travaux sous tension "; un grand nombre d'agents travaillent, en eff normalement sur le réseau électrique sans que le courant ne soit coupé, il faut donc que tous leurs gestes soient faits en pensant au risque d'électrisation ou d'électrocution.

Dans le cas des travaux sous rayonnements ionisants, il faut également que les interventions des agents en zone contrôlée prennent en compte le risque d'irradiation, au niveau de la préparation et de l'exécution; c'est cet apprentissage des gestes du métier, face à un risque imperceptible, qui constitue un aspect essentiel de la formation en radioprotection.

« Tout agent travaillant dans une centrale nucléaiı, à quelqu * niveau qu'il soit, est concerné par sa propre protection. "

Il n'est, dès lors, pas question, une fois ce principe admis, de concevoir un plan de formation du personnel dans lequel la radioprotection resterait l'apanage de quelques spécialistes, en nombre limité, sortes de " grands prêtres » du nucléaire, guidant des travailleurs ignorants et distribuant parcimonieusement des bribes de leur savoir au travers des zones contrôlées. La réglementation a d'ailleurs défini clairement, dans l'article 11 du décret 75-306 du 28 avril 1975, les obligations de l'employeur en matière de formation et d'information.

« L'employeur doit préparer au travail sous rayonnements tout travailleur affecté à y travailler occasionnellement et l'informer, par une notice appropriée dont la remise donne lieu à émargement de l'intéressé :

voL. $15-\mathrm{N}^{\circ} 4$ 
- des risques d'irradiation ou de contamination auxquels son travail est susceptible de l'exposer;

- des précautions à prendre pour éviter ces risques;

- des méthodes de travail offrant les meilleures garanties de sécurité;

- des garanties que comportent pour lui les mesures physiques et les examens médicaux périodiques.

Cette formation et cette information doivent être adaptées à la nature des travaux effectués et aux travailleurs auxquels elles s'adressent ».

E.D.F. se doit donc de former tous ses agents qui sont amenés, par leurs эctivités, à travailler en zone contrôlée.

Remarque. - On oppose quelquefois, en matière de radioprotection, les installations industrielles aux centres de recherche; le terme d' " autoprotection " a été utilisé abusivement pour définir le principe d'organisation mis en place par E.D.F. dans les centrales, et il a été ainsi opposé à une doctrine plus rigide adoptée parfois dans les laboratoires et les centres d'études.

Il n'y a pas de réelle incompatibilité entre ces deux principes, la sécurité restant l'affaire de tous, chacun devant être conscient des risques qu'il court et fait courir aux autres dans son travail. Les centres de recherches, notamment au C.E.A., ont eu à découvrir les problèmes et à définir, pour chacun d'eux, les méthodes et techniques de protection. Il leur a fallu disposer de moyens spécifiques et imposer une discipline rigoureuse.

L'ind ustrie, et en particulier E.D.F., profite largement de cette expérience, notamment dans le domaine de la connaissance des risques; la protection individuelle n'exclut pas le recours à une surveillance et à une assistance par des services spécialisés, imposés d'ailleurs par la réglementation (article 8 du décret cité plus haut). A Électricité de France, cette double disposition est bien assurée, les agenis d'exploitation étant assistés et contrôlés par une section spécialisée, propre à chaque centrale. Le Service central de la Production thermique dispose en plus d'un département chargé d'élaborer la doctrine et de définir les moyens permettant de la respecter.

\subsection{LA FORMATION DES AGENTS D'ENTREPRISES EXTÉRIEURES}

Tout comme le personnel E.D.F., les agents d'entreprises extérieures intervenant en zone contrôlée doivent avoir reçu une formation adaptée à leur activité; l'enseignement est assuré soit par l'employeur lui-même (cas général des grosses entreprises), soit par des organismes spécialisés (A.P.A.V.E., A.I.N.F., S.I.F.O.P., G.I.F.O.P. $\left({ }^{2}\right)$ et le C.E.A. et ses filiales).

Le décret 77-1321 du 29 novembre 1977 a, par ailleurs, défini un certain nombre d'obligations pour E.D.F. vis-à-vis des entreprises extérieures, en

(2) A.P.A.V.E., Association de propriétaires d'appareils à vapeur et électriques. A.I.N.F., Association interprofessionnelle de France pour la prévention des accidents et de l'incendie. S.I.F.O.P., Service interconsulaire de formation et de perfectionnement (Nord). G.I.F.O.P., Groupement interprofessionnel de formation, d'orientation et de perfectionnement (Est). 
matière de sécurité et radioprotection; elles se traduisent par une information sur les consignes de radioprotection de la centrale, et la remise au responsable de ces entreprises du " guide des entreprises prestataires " qui rappelle les règles en matière de radioprotection et qui fixe les conditions minimales d'intervention en zone contrôlée.

\section{ORGANISATION DE LA FORMATION EN RADIOPROTECTION A E.D.F.}

\subsection{Formation DE BASE (tableau II)}

A Électricité de France, chaque agent travaillant en zone contrôlée reçoit une « habilitation " en radioprotection; pour obtenir cette " habilitation " délivrée par le chef de centrale, une formation adaptée à la fonction qu'il occupe est requise; deux niveaux de formation ont été ainsi définis :

- le "premier palier " (habilitation RP 1), destiné à l'ensemble des agents, doit leur permettre de faire face à tous les problèmes courants qui se posent à eux en zone contrôlée;

- le " deuxième palier " (habilitation RP 2), destiné aux chefs de travaux, leur donne la formation nécessaire pour faire face à leur responsabilité sur le plan collectif.

\section{TABLEAU II}

FORMATION DE BASE

\begin{tabular}{|c|c|c|}
\hline Nature du stage & Personnel intéressé & Durée \\
\cline { 1 - 3 } $\begin{array}{c}\text { Premier palier (RP 1) ......... } \\
\text { Deuxième palier (RP 2)......... }\end{array}$ & $\begin{array}{c}\text { Tous agents affectés } \\
\text { en zone contrôlée } \\
\text { Chefs de travaux en zone contrôlée }\end{array}$ & 1 semaine \\
\cline { 1 - 3 }
\end{tabular}

\subsection{LA FORMATION SPÉCIALISÉE (tableau III)}

Comparés aux efforts déployés pour l'acquisition, par l'ensemble des agents travaillant dans les centrales nucléaires, du niveau minimal rəquis (RP 1 ou RP 2), les problèmes posés par la formation de "spécialistes 》 (radiochimistes, techniciens en radioprotection, techniciens de contrôle de l'environnement, etc.) sont nettement moins ardus, ces " spécialistes " étant, dans les centrales nucléaires, en nombre relativement limité. Quant à la formation du personnel d'encadrement, elle est plutôt assimilable à ure " information » et à une prise de conscience d'un paramètre nouveau qu'à une " reconversion au nucléaire ».

Contrairement à celle des premier et deuxième paliers qui sont délivrés sur les sites des centrales nucléaires, cette formation complémentaire se

voL. $15-\mathrm{N}^{\circ} 4$ 
TABLEAU III

STAGES ORgANisÉS SUR LE PLAN NATIONAL

\begin{tabular}{|c|c|c|}
\hline Nature du stage & Personnel intéressé & Durée \\
\hline $\begin{array}{l}\text { Formation en radioprotection... } \\
\text { Connaissance de base en radio- } \\
\text { protection .................. } \\
\text { Préparation des travaux d'entre- } \\
\text { tien ....................... } \\
\text { Mesures de radioprotection au } \\
\text { service technique............ } \\
\text { Mesures pour la radioprotection } \\
\text { du personnel ............... } \\
\text { Conception des installations.... }\end{array}$ & $\begin{array}{c}\text { Techniciens et cadres } \\
\text { Tous les ingénieurs } \\
\text { Préparateurs des services entretien } \\
\text { Techniciens des services techniques } \\
\text { Techniciens des sections } \\
\text { sécurité-radioprotection } \\
\text { Projeteurs }\end{array}$ & $\begin{array}{c}1 \text { semaine } \\
2 \text { semaines } \\
4 \text { jours } \\
2 \text { semaines } \\
1 \text { semaine } \\
1 \text { semaine } \\
\text { et demie }\end{array}$ \\
\hline
\end{tabular}

présente sous forme de stages organisés essentiellement par le Département de Radioprotection du Service de la Production thermique, et qui sont destinés:

- à donner une formation technique " spécialisée " aux techniciens des sections de radioprotection et des laboratoires de contrôle des effluents et de l'environnement $\left({ }^{3}\right)$;

- à sensibiliser les préparateurs, afin de leur faire prendre en considération la radioprotection dès la préparation d'une intervention;

- à informer et à recycler les cadres et ingénieurs des centrales nucléaires sur l'évolution de la réglementation, sur les techniques nouvelles, etc.);

- à qualifier les " formateurs " parmi les techniciens et les cadres des centrales;

- à sensibiliser les dessinateurs, projeteurs et ingénieurs des bureaux d'étude, afin d'améliorer la conception des nouvelles installations.

\section{LE CONTENU DE LA FORMATION EN RADIOPROTECTION A E.D.F.}

Il ne sera envisagé ici que la formation de base; les stages destinés au personnel spécialisé sont, nous l'avons vu, plutôt orientés vers l'acquisition de techniques particulières.

\subsection{UNE FORMATION PRAGMATIQUE}

En matière de radioprotection, l'essentiel de la formation doit être axé sur l'enseignement d'un certain nombre de réflexes visant un but unique : la réduction de l'exposition des travailleurs aux rayonnements ionisants.

(3) La formation de ces derniers est complétée, conformément à la réglementation, par un stage au Service central de protection contre les rayonnements ionisants (S.C.P.R.I.). 
La formation doit donc être pragmatique; la partie consacrée à l'acquisition de connaissances théoriques est réduite au minimum; notamment, les premier et deuxième paliers ne font pas appel à des notions de base dépassant le niveau de l'arithmétique élémentaire; en effet, outre le fait d'être totalement incompréhensibles pour la plupart, les formules mathématiques ou les symboles physiques ont des conséquences psychologiques néfastes sur les agents, car elles les confortent dans l'idée - communément admise au départ - que la radioprotection est une sorte d'alchimie secrète réservée aux initiés; ils auront alors tendance à se sentir dégagés de toutes responsabilités, et supporteront difficilement des contraintes de radioprotection imposées par d'autres, notamment par les agents des sections Sécuritéradioprotection.

La radioprotection est, avant tout, dans les centrales nucléaires, une question de bon sens, de soin et d'ordre dans le travail; de même qu'on peut changer un fusible en toute sécurité sans connaître à fond les lois de Kirchoff, la connaissance des schémas de désintégration des radioéléments n'est pas indispensable pour réparer une vanne ou une pompe en milieu irradiant.

\subsection{LES PROGRAMMES DE FORMATION RP 1 ET RP 2}

\section{Niveau RP 1}

Les agents doivent, à l'issue de cette formation d'une durée de 40 heures :

les balisages;

- pouvoir circuler en toute sécurité dans les zones nucléaires en respectant

- exécuter sans « erreur " le travail qui leur est confié;

- savoir utiliser les moyens de protection individuelle;

- être capables de se contrôler (vis-à-vis de la contamination).

Les thèmes abordés sont notamment :

- les caractéristiques du travail en centrale nucléaire;

- la notion de dose;

- la notion de débit de dose;

- les modes d'irradiation (irradiation externe/contamination) et les protections correspondantes;

- la nature des mesures de radioprotection;

- la pratique d'utilisation des moyens de protection;

- les techniques de mesures.

Enfin un « chantier école ", reconstitution aussi exacte que possible d'un chantier nucléaire, permet de préparer les agents à la réalité d'une intervention en zone contrôlée.

\section{Niveau RP 2}

Cette formation, également d'une durée de 40 heures, donne aux chefs de travaux l'aptitude à diriger une équipe de travail en zone contrôlée, dans 
le cadre d'une préparation, et à utiliser les résultats des appareils de protection collectifs. Elle est axée sur les thèmes suivants :

- rappel et vérification de l'acquis du premier palier;

- irradiation externe;

- contamination atmosphérique;

- contamination surfacique.

Ces thèmes sont essentiels pour préparer et exécuter les interventions.

Trois séances sont consacrées au " chantier école "; toutes les phases d'un chantier en zone contrôlée sont examinées en détail, depuis l'établissement de la " cartographie » des lieux jusqu'au repli final du chantier.

\subsection{LES SUPPORTS MATÉRIELS}

Des dossiers pédagogiques ont été élaborés pour les niveaux RP 1 et RP 2 et pour certains stages; ils regroupent, pour chacun des thèmes abordés, un guide de séance, des aides pédagogiques (tableaux, diapositives, etc.) et des documents à remettre aux stagiaires, et constituent ainsi une aide aux animateurs.

Ces dossiers servent également de " guides " à certaines entreprises extérieures assurant la formation de leurs propres agents, ainsi qu'aux organismes spécialisés dans cette formation; ils permettent de garantir une certaine homogénéité entre les niveaux de formation des agents appartenant à diverses entreprises.

En outre, deux documents ont été établis par E.D.F. et servent de base à la radioprotection dans les centrales nucléaires, il s'agit :

- du Carnet de radioprotection qui fixe les prescriptions en matière de radioprotection; il est distribué à tous les agents;

- du Guide pratique de la radioprotection qui constitue un recueil de recommandations et s'adresse particulièrement au personnel d'encadrement.

\section{CONTRÔLE DE QUALITÉ DE LA FORMATION EN RADIOPROTECTION}

\subsection{LE CONTRÔLE DES CONNAISSANCES}

L'habilitation en radioprotection n'est délivrée, à l'issue de la période de formation, qu'après un contrôle qui s'exerce sous forme de "tests " auxquels sont soumis les agents d'entreprises extérieures comme les agents E.D.F., avant l'entrée en zone contrôlée; la sélection est particulièrement stricte pour les agents du niveau RP 2 (chefs de chantier).

Ce contrôle est indispensable pour garantir la bonne compréhension et l'assimilation par les agents des notions de base en radioprotection; il 
permet, en outre, de vérifier l'aptitude des formateurs à dispenser les connaissances avec les moyens pédagogiques mis à leur disposition.

\subsection{LA VALIDITÉ DU PLAN DE FORMATION D'E.D.F.}

Si le " contrôle des connaissances " donne une certaine crédibilité à l'habilitation en radioprotection, en revanche, il ne garantit pas que la formation telle qu'elle est conçue actuellement, réponde aux objectifs poursuivis; seuls les résultats obtenus in situ, sur les tranches nucléaires en exploitation, notamment pendant les périodes d'arrêt de tranche, donnent le verdict final sur la formation du personnel en radioprotection; à cet égard, le déroulement satisfaisant des opérations effectuées lors des premiers rechargements des réacteurs PWR de $900 \mathrm{MW}$ devrait démontrer la validité du concept d'une radioprotection simple, pratique, intégrée au travail, et utilisable par tous.

\section{DONNÉES ÉCONOMIQUES SUR LA FORMATION EN RADIOPROTECTION}

\subsection{LE COÛT DE LA FORMATION}

Il paraît utile d'apporter quelques données économiques sur cet investissement particulier que représente la formation en radioprotection à E.D.F.; son coût est estimé, actuellement, à une valeur comprise entre 300 et $500 \mathrm{~F}$ par jour $\times$ agent pour la « prestation enseignement " proprement dite, hormis les coûts " annexes » (hébergement, etc.) et le manque à gagner dû à l'absence de l'agent à son poste de travail.

En 1979, les stages de formation ont été suivis par 4600 agents, ce qui représente un nombre de jours total de 9800 et un coût global de 5 millions de francs. Ce coût doit être largement majoré si l'on tient compte des actions de formation permanente exercées notamment par les contremaîtres et les chefs de travaux dans le cadre d'un travail déterminé.

\subsection{LA FORMATION ACTUELLE EST-ELLE RENTABLE?}

En matière d'investissements, en vue de diminuer les doses du personnel, certains parlent du " coût de l'homme-rem » et justifient, grâce à cette notion, l'engagement — ou le non-engagement — des dépenses.

Si la relation coût/bénéfice ne peut être mesurée avec précision en ce qui concerne les investissements « matériels ", elle est encore plus difficilement appréciable pour cet investissement particulier que représente la formation : à défaut de pouvoir chiffrer les gains escomptés, on peut cependant affirmer que le manque de formation des agents aurait des conséquences fâcheuses sur les doses du personnel; en effet :

- la formation actuelle permet, avant tout, d'éviter les erreurs grossières qui " coûteraient " cher en homme-rems, voire conduiraient à de réels accidents; ce point est tout à fait capital; 
- elle évite précisément le gaspillage des investissements que l'on consent par ailleurs, notamment en matière d'outillages perfectionnés ou de modifications d'installations;

- elle permet également de "valoriser " l'homme-rem, en limitant le nombre d'agents par opération; le manque de formation des agents rendrait indispensable la présence sur les chantiers, d'un ou de plusieurs anges gardiens, contrôlant faits et gestes de chaque opérateur et recevant, par la même occasion, des doses équivalentes.

\section{CONCLUSION}

La nécessité absolue de diminuer l'exposition des travailleurs aux rayonnements ionisants explique l'ampleur du programme de formation en radioprotection des agents des centrales nucléaires. L'orientation donnée à l'enseignement de la radioprotection à E.D.F. répond à ce souci de réduction des doses.

La radioprotection ne doit plus être considérée comme une science « à part » mais s'intégrer davantage à la formation professionnelle en général : savoir travailler en milieu radioactif, c'est d'abord travailler vite et bien. $\mathrm{La}$ formation du personnel est un maillon essentiel dans cette recherche de la qualité et de la sécurité dans le travail; cette recherche exige, en outre, la participation active des Comités d'hygiène et de sécurité et de la Médecine du travail. 